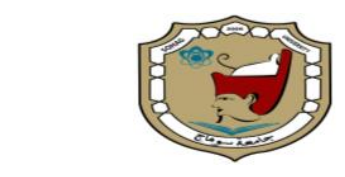

\title{
Cancer-Related Signals of Epithelial Cell Adhesion Molecule (EpCAM): Review
}

\author{
Sheren FM Ahmed*, Nagwa Abd El-Sadek Ahmed*, Kamal A A \\ Hassanein**, and Eman Muhammad Salah El-Deen Muhammad*. \\ * Department of Pathology, Faculty of Medicine, Sohag University, Egypt. \\ ** Department of General Surgery, Faculty of Medicine, Sohag University, Egypt.
}

\begin{abstract}
Background and Aim: EpCAM, also known as cluster of differentiation 326 (CD326), is a glycosylated, type I trans-membrane molecule, it is overexpressed in several types of neoplasms such as colorectal cancer and breast cancer. It is primarily identified as one of cell adhesion molecules (CAMs). Recently, it is considered as an emerging marker for detection of cancer stem cells (CSCs) in various neoplasms. EpCAM is proved as a critical factor in carcinogenesis. Emerging evidences suggest that it may play an important role in tumor development and progression; also it is reported to be implicated in tumor invasion and metastasis. The aim of this review was to highlight the current knowledge on the structure and function of EpCAM focusing on some mechanisms that may be involved in cancer progression with emphasis on the clinical significance of EpCAM as a diagnostic and prognostic marker.
\end{abstract}

Conclusion: EpCAM molecule is a prognostic indicator referring to tumor progression, metastasis and relapse. Targeting EpCAM in therapeutic approaches may be a useful strategy for cancer treatment and prevention of metastasis and relapse.

Keywords: EpCAM, CSCS, CAMs, Tumor progression, Invasion

\section{Introduction}

EpCAM is a $40 \mathrm{kDa}$ glycosylated, type I trans-membrane molecule. It is encoded by TACSTD1 gene which is located on chromosome 2 ( $2 \mathrm{p} 21)$. It is mainly expressed on normal epithelial cells and embryonic stem cells. EpCA$\mathrm{M}$ molecule exists on the basolateral surface in most of normal epithelial tissues mediating cell-cell adhesion. EpCAM expression increases in epithelial benign and malignant tumors [1].

\section{Structure}

EpCAM consists of 314 amino acids. Structurally, the full-length EpCAM protein can be divided into four essential parts. The first part is a stretch of signal peptide consists of 23 amino acids located at the $\mathrm{N}$-terminal of EpCAM that is cleaved off during synthesis. Therefore, the amino acid sequence for a mature EpCAM protein starts only at Glutamine 24 (Gln24). The other three parts constitute the main domains of EpCAM molecule (Figure1) and include; an extracellular domain (EpEX), trans-membrane domain, and an intracellular domain (EpICD). EpEX domain is composed of 242 amino acids and it has three independent glycosylation sites and cysteine residues. EpEX domain is divided into 3 subdomains; the first one has 2 epithelial growth factor binding sites, the second one has IGF and thyroglobulin 
binding proteins and the last subdomain is cysteine-free. On the other hand, the small intracellular domain (EpICD) consists of 26 amino acids and has two tyrosine and actinin binding sites. The single trans-membrane portion consists of 23 amino acids [2\&3].

\section{EpCAM Expression in Healthy \\ Tissues}

EpCAM exists on the lateral and basal membranes in most of normal epithelial tissues mediating cell-cell adhesion. In most of epithelial tissues, strong EpCAM signals mainly localized to the basolateral surface were obtained by IHC, while no EpCAM signals could be detected in non-epithelial tissues. EpCAM shows different expression levels between the different tissues and different types of cells. High EpCAM levels were mostly detected in the normal epithelia of colon and endometrium. It was reported that EpCAM showed lower expression levels in normal epithelial tissues compared to its expression levels within the neoplastic cells [4].

\section{Function}

\section{- In cell-cell adhesions}

The tissue architecture is mainly maintained by mean of cell-cell adhesive interactions. EpCAM doesn't structurally belong to any of the major CAMs families, although it is primarily identified as one of adhesion molecules. It interacts with other adhesion molecules such as E-Cadherin and Claudins1\&7. EpCAM may enhance cell-cell adhesion through intercellular trans-oligomers formation. It also regulates adhesive structures between cell to cell and cell to matrix. On the molecular level, it interacts with two adhesion molecules (claudin1\&7) preventing their degradation and regulating their function in tight junctions [5].

\section{- In cell proliferation}

EpCAM plays an important regulating role in cellular proliferation that is based on its reactivity in healthy tissuees where it is preferentially localized to the proliferative areas. Additional evidence for its role in regulating the cell proliferation is based on the relationnship between cell proliferation and its expression levels [6].

\section{EpCAM Cleavage and Downstream Signalization}

It was reported that EpCAM is mainly cleaved by TNF- $\alpha$ Converting Enzyme (TACE). Regulated intra-membrane proteolysis (RIP) mediates EpCAM cleavage and results in release of EpEX and EpICD domains. EpIC has a central role in EpCAM downstream signalization. Cellular contacts binding of EpEX domain results in activation of EpCAM cleavage and downstream signaling that is mediated by metalloproteinase and disintegrin. The first cleavage is mediated by disintegrin and metalloproteinase17 (ADAM17), also called TACE, results in liberation of the extracellular domain (EpEX) in surrounding environment. RIP is also triggered by cell-cell contact. The second cleavage is mediated by $\gamma$-secretase, releasing the intercellular EpIC in the cytosol. EpICD binds to $\beta$ catenin and four and half LIM domains protein 2 (FHL2) forming a complex that shuttles into the nucleus, and activeetes cell division by promoting the expression of c-Myc and cyclin genes that are responsible for cell proliferation [7].

\section{EpCAM Expression in Cancer}

EpCAM is proved as a critical factor in the process of carcinogenesis (Figure2), but there is no clear knowledge on its exact biological roles in tumor development and progression. Its expression level varies depending on numerous parameters. EpCAM is frequently overexpressed in most of epithelial 
tumors, but it is usually absent in tumors of non-epithelial origin such as sarcomas and lympho-mas. EpCAM distribution varies accor-ding to carcinoma type. Its expression is localized mainly to the cell memb-rane, also it was reported that EpCAM signals could be detected in the cytopl-asm and nucleus, since it is subject to RIP and cleavage. Its role in cancer develo-ppment and progression is likely related to its subcellular redistribution as well as its expression level [8\&9].

\section{Role in intercellular adhesions}

EpCAM molecule can regulate the intercellular adhesive interactions and modulate their strength; it interacts with other adhesion molecules regulating their function. EpCAM up-regulation in carcinomas has a negative effect on the strength of cell adhesions. This effect is mediated by its interacttion with E-Cadherin that leads to loosening of cell-cell adhesion. EpCAM mediates the disruption of the link between $\alpha$-catenin and $F$-actin. On the other hand, EpCAM down-regulation is followed by an increase in cellular $\alpha$ catenin that promotes the adhesion of E-cadherin/Catenin complex to the cytoskeleton. EpCAM can modify the composition and functions of tight junctions by regulating the action of claudins. Knockdown of EpCAM reduces claudin- $1 \& 7$ resulting in increase of the tight junction between cells that consequently increases the cellular resistance to disseminate, that indicates the critical role of EpCAM in cancer propagation and metastasis as it has an important role in the onset of invasion [10].

\section{- Role in cell proliferation}

EpCAM expression correlates with proliferation state of most of epithelial cells, its expression is usually enhanced in less differentiated areas. EpIC has an important role in cell proliferation and constitutes the active signa- ling intracellular portion. EpCAM controls cell proliferation via regulation of cyclin D1 that is the key player at the transcriptional level. Recent findings suggest that EpEX acts as ligand for epidermal growth factor receptor (EGFR) maintaining cell proliferation by promoting cell signalization through ERK1/2 and AKT [11].

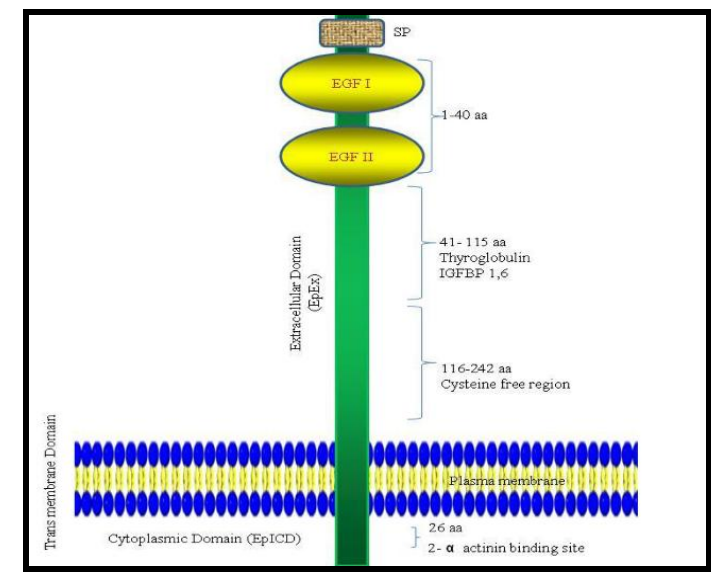

Figure (1): Schematic diagram of EpCAM structure [3].

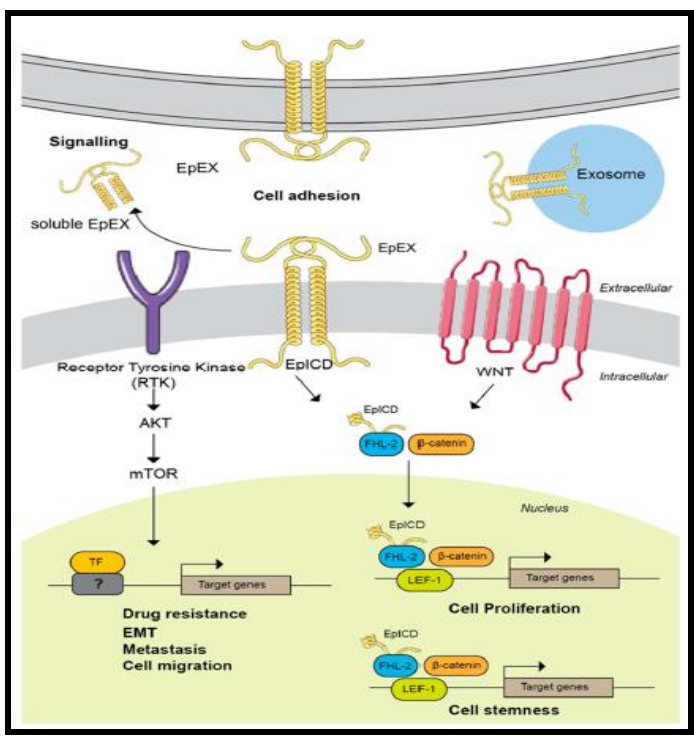

Figure (2): EpCAM localization and modulation of its downstream targets in cancer [8].

- EрCAM and epithelial-to-mesenchymal transition (EMT)

Biological regulation of EpCAM expression levels during EMT process is a matter of controversy. EMT is defined as a complex process that is important during progression of malignant tumors and critical for their ability to 
invade and to colonize in other parts of the body. EMT can modulate the adhesion molecules that are expressed by the cells with acquiring a migratory and invasive behavior. The cells during EMT show high elasticity. They show a spectrum of intermediate phases between epithelial and mesenchymal states [12].

\section{- Role of EpCAM as stem cell marker}

CSCs are group of cells that are characterized by their ability to selfrenewal and differentiation. CSCs have high levels of pro-invasive genes and enhance the tumor progression and invasion. CSCs usually have high invasive and metastatic potential. EpCAM is reported to be one of stem cell markers. It is expressed in various stem, precursor cells, and CSCs. So, it has an important role in cancer progression. EpCAM has a critical role in promotion and maintenance of proliferation and differentiation of CSCs and neoplastic cells. It also promotes the survival of neoplastic cells at metastatic sites [13].

\section{- EpCAM role in invasion and migration}

Meanwhile, EpCAM is considered as one of adhesion molecules; it can promote the cellular mobility. Emerging findings suggest that EpCAM molecule is an important factor involved in tumor development and progression. EpCAM is critical factor in regulation of tumor invasion and metastasis. Knockdown of EpCAM levels leads to loosening of adhesions between the neoplastic cells and leads to their propagation and migration. However, the role of EpCAM in tumor progression and invasion seems to be context dependent [14].

\section{- EpCAM expression on circulating tumor cells (CTCs)}

There is no clear information about EpCAM expression on CTCs. CTCs are liberated from the primary tumor then enter the blood stream and have a critical role in the metastatic process. CTCs level is considered as a prongostic factor for various types of cancers and considered as a major challenge in cancer therapy. The detection and isolation of CTCs is of major importance in clinical practice, measuring variations in EpCAM expression levels has great clinical significance as indicator for the onset of cancer invasion. EpCAM expression levels in serum are considered as indicator for tumor progression toward invasion and metastasis. It has a major role as one of the newest and the most popular markers in oncology field. Hence, evaluation of EpCAM expression is considered as a novel approach in clinical trials for cancer therapeutic strategies [15].

\section{Clinical Significance}

\section{Role in therapeutics}

EpCAM is involved in cell proliferation, tumor progression, invasion and spread. It has attracted great attention as an emerging target for antibodybased cancer immunotherapy. Several clinical approaches targeting EpCAM in different therapeutic strategies were undertaken over years. EpCAM also implicated in chemoresistance and relapse. So, targeting EpCAM in cancer therapy can help to achieve effective results with chemotherapy in treatment of malignant tumors. Knockdown of EpCAM shows suppressed proliferation, enhanced chemosensitivity and radiosensitivity, and decreased relapse. EpCAM expression regulates the stemness which can be used in clinical practice as a new strategy of anticancer therapy [16].

\section{Diagnostic role}

EpCAM molecule is used as a diagnostic marker to differentiate tumors with histopathological resemblance; it was 
used to distinguish hepatocellular carcinoma (HCC) from similar neoplasms such as, metastatic adenocarcinoma and cholangiocarcinoma. The pattern of EpCAM expression in HCC is more homogeneous and diffused with intense cytoplasmic and/or membraneous staining. Also, it was reported that basal cell carcinoma with squamous metaplasia showed diffuse EpCAM overexpression, while basaloid squamous cell carcinoma showed low and sporadic reactivity [17\&18].

\section{Prognostic role}

In efforts to clarify the relationship between EpCAM expression in various neoplasms and the prognosis, many studies were arising. Expression of EpCAM and its association with outcome and prognosis have been a matter of controversy. The role of EpCAM molecule as prognostic factor is dependent mainly on cancer type. EpCAM overexpression is associated with good prognosis and improved survival in some neoplasms such as, thyroid carcinoma and gastric carcinoma. Meanwhile, in other carcinoma types like pancreatic and nasopharyngeal carcinoma; it is a poor prognostic indicator referring to tumor progression, metastasis and relapse where it is associated with worse prognosis and decreased survival. Interestingly, in colorectal carcinoma and breast carcinoma both effects have been reported. Surveillance of EpCAM levels can help in identifying patients with high risk of disease recurrence or progression [19].

\section{CONCLUSION}

EpCAM molecule is a prognostic indicator referring to tumor progression, metastasis and relapse. Targeting EpCAM in therapeutic approaches may be a useful strategy for cancer treatment and prevention of metastasis and relapse.

\section{LIST OF ABBRIVATIONS}

CAMs: Cell adhesion molecules, CD: Cluster of differentiation, CSCs: Cancer stem cells, CTCs: Circulating tumor cells, EpCAM: Epithelial Cell Adhesion Molecule, EpEX: Extracellular domain of EpCAM, EpICD: Intracellular domain of EpCAM, Gln24: Glutamine 24, HCC: Hepatocellular carcinoma, IGF: Insulin- growth factor, LIM domains: Zinc-binding domain family and are named after their initial discovery in the three proteins (LIN-11, Is11 and MEC-3). RIP: Intramembrane proteolysis, TACE: TNF- $\alpha$ Converting Enzyme.

\section{REFERENCES}

1. Schnell U, Cirulli V and Giepmans: EpCAM structure and function in health and disease. Biochemica and Biophysica Acta. 2013; 1828: 19892001.

2. Pavsic M, Guncar G, Djinovic-Carugo $\mathrm{K}$ and Lenarcic B: Crystal structure and its bearing towards an understanding of key biological functions of EpCAM. National journal of Community. 2014; 5: 4764-4773.

3. Vasanthakumar S, Sasikala P, Padma M, Balachandar V, Venkatesh B and Ganesan S: EpCAM as a novel therapeutic target for hepatocellular carcinoma. Journal of Oncological Sciences. 2017; 3: 71-76.

4. Keller L, Werner $\mathrm{S}$ and Pantel $\mathrm{K}$ : Biology and clinical relevance of EpCAM. Cell Stress. 2019; 3: 165-180.

5. Gaber A, Kim S, Kaake R, Bencina M, Krogan N, Sali A, Pavsic $M$ and Lenarcic B: EpCAM homooligomerization is not the basis for its role in cell-cell adhesion. Scientific Reports. 2018; 8: 13269-13278.

6. Liang K, Tso H, Hung S, Kuan I, Lai J, Ke F, Chuang Y, Liu I, Wang Y, Chen $\mathrm{R}$ and $\mathrm{Wu} \mathrm{H}$.: Extracellular domain of EpCAM enhances tumor progression through EGFR signaling in cancer cells. Cancer Letters. 2018; 433: 165175.

7. Tsaktanis $\mathrm{T}$, Kremling $\mathrm{H}$, Pavsic M, von Stackelberg R, Mack B, Fukumori 
A, Steiner H, Vielmuth F, Spindler V, Huang Z, Jakubowski J, Stoecklein N, Luxenburger E, Lauber K, Lenarcic B and Gires O: Cleavage and cell adhesion properties of human epithelial cell adhesion molecule. Journal of Biological Chemistry. 2015; 290: 24574-24591.

8. Mohtar M, Syafruddin S, Nasir S and LowT: Revisiting the Roles of ProMetastatic EpCAM in Cancer. Biomolecules. 2020; 10: 255.

9. Yahyazadeh-Mashhadi S, Kazemimanesh M, Arashkia A, Azadmanesh K, Meshkat Z, Golichenari B and Sahebkar A: Shedding light on the EpCAM: An overview. Journal of Cell Physiology. 2019; 234:12569-12580.

10.Huang L, Yang Y, Yang F, Liu S, Zhu $Z$, Lei $Z$ and Guo J: Functions of EpCAM in physiological processes and diseases: Review. International Journal of Molecular Medicine. 2018; 42: 1771-1785.

11. Wang M, Sun R, Zhou X, Zhang M, Lu J, Yang Y, Zeng L, Yang X, Shi L, Xiao R, Wang $\mathrm{H}$ and Mai S: Epithelial cell adhesion molecule overexpression regulates epithelial-mesenchymal transition, stemness and metastasis of carcinoma cells via the PTEN/AKT/mTOR pathway. Cell Death Diseases. 2018; 9: 2-15.

12. Nieto $M$, Huang $R$, Jackson $R$ and Thiery J: EMT. Cell. 2016; 166: 21-45.

13. Sato R, Semba T, Saya H and Arima Y: Stem Cells and EpithelialMesenchymal Transition in Cancer: Biological Implications and Therapeutic Targets. Stem Cells. 2016; 34: 1997-2007.
14.Wang $\mathrm{P}$, Weng $\mathrm{C}$, Hou Y, Jian S, Fang $\mathrm{K}$, Hou $\mathrm{M}$ and Cheng K: Activation of VCAM-1 and its associated molecule CD44 leads to increased malignant potential of breast cancer cells. International Journal of Molecular Science. 2014; 15: 3560-3579.

15.Ring A, Mineyev N, Zhu W, Park E, Lomas C, Punj V, Yu M , Barrak D, Forte V, Porras T, Debu Tripathy D and Lang J: EpCAM based capture detects and recovers circulating tumor cells from all subtypes of breast cancer except claudin-low. Oncotarget. 2015; 6(42): 44623.

16. Vasanthakumar S, Sasikala P, Padma M, Balachandar V, Venkatesh B and Ganesan S: EpCAM as a novel therapeutic target for hepatocellular carcinoma. Journal of Oncological Sciences. 2017; 3: 71-76.

17.Karabork A, Kaygusuz G and Ekinci C: The best immunohistochemical panel for differentiating hepatocellular carcinoma from metastatic adenocarcinoma. Pathology Research and Practice. 2010; 206: 572-577.

18. Webb D, Mentrikoski M, Verduin L, Brill L and Wick M: Basal cell carcinoma vs basaloid squamous cell carcinoma of the skin: An immunohistochemical reappraisal. Annals of Diagnostic Pathology. 2015; 19: 70-75.

19.Herreros-Pomares A, Aguilar-Gallardo C, Calabuig-Farinas S, Sirera R, Jantus-Lewintre $\mathrm{E}$ and Camps C: EpCAM duality becomes this molecule in a new Dr. Jekyll and Mr. Hyde tale. Critical Reviews in Oncology/Hematology. 2018; 126: 5263. 